\title{
Sweet collaborations
}

If the field of glycomics had to be described in two words, those words would be complexity and collaboration. The system-wide study of carbohydrates and their functions presents unique challenges that position the field right at the crossroads of many different disciplines. In this issue, we present two important new methods for glycomics researchers and a review underscoring the need for concerted efforts from different areas of biology, chemistry and bioinformatics.

Glycomics is a relatively young field, but what it lacks in age it makes up in complexity. For example, carbohydrates, also known as glycans, are synthesized in a nontemplate-driven process by hundreds of enzymes, making them very diverse in both composition and structure. The attachment of glycans to proteins is the most common post-translational modification, and one protein can have many glycosylation sites, each site harboring different glycans. These glycans on the glycoproteins in turn can interact with carbohydrate binding proteins, the so-called lectins that specifically recognize distinct carbohydrate structures and whose function is often determined by the interactions with glycans.

On one side, this high degree of intricacy and specificity allows for very precise regulation in processes such as immune responses, cell differentiation and migration. On the flip side, aberrantly glycosylated proteins are involved in cancer, and pathogens exploit the system to evade immune surveillance. Discovering the function of glycans is thus not only important to understand basic biology, but also to explain disease mechanisms.

To fully understand and characterize all the players involved, a whole battery of methodologies and expertise is needed: biochemical approaches can identify the glycans that interact with carbohydrate binding proteins, information about the structure of these glycans can lead to the identification of enzymes needed for their synthesis, which in turn can drive a genetic approach.

In each of these areas the development of new tools and techniques is critical for the advancement of the field. And scientists interested in the intricacies of glycan functions quickly realized that only the pooling of expertise will give them the means to tackle a problem of this magnitude. On page 817, Ram Sasisekharan, an investigator instrumental in developing the bioinformatics tools for the Consortium for Functional
Glycomics, reviews approaches undertaken by members of several consortia to integrate data from different disciplines to elucidate the function of glycans in vivo.

A noteworthy aspect of these consortia is that they were created right at the emergence of the field of glycomics. Also, collaborations do not stop at the borders of individual consortia; rather their members seek to create a world-wide net of researchers working on different aspects of glycomics. Many scientists are members of more than one international consortium; some investigators in the Consortium for Functional Glycomics, for example, are also on the executive committee of the Japanese Human disease glycomics/proteome initiative and participate in EuroCarb, an initiative that seeks to connect all the carbohydrate structure databases in the world.

Many of these initiatives are still young or even in their infancy, and are largely funded by government grants. They have set milestones, so their success can be measured at the end of the funding period, and the skeptics who argued that the grant money would have been better spent supporting individual laboratories may be convinced otherwise. One milestone is the creation of new tools that could not have easily been generated by individual efforts. Good examples are core facilities already set up by several consortia that generate essential resources such as transgenic mice or glycan arrays. But the availability of these resources in itself is not enough; another measure of success will be how they are adopted by other scientists who will use these tools and in turn move the field forward.

Despite the progress glycomics is already making, a healthy realism prevails. James Paulson, director of the Consortium for Functional Glycomics says that their goal is not to elucidate the whole glycome, a prohibitively huge task, but to accelerate the rate at which information is acquired and to use this to define new paradigms for carbohydrate binding proteins.

Such realism does not take away from the enthusiasm with which this field collectively tackles difficult questions. This is an appealing feature that ideally should describe any scientific endeavor; in other fields, however, competition has been known to outweigh collaboration. It will be interesting to follow the development of the glycomics community to see if the tight networking continues and meets its goals - if it does, this field may provide not only tools and resources, but also a model of science at its best. 